\title{
Genome mapping and characterization of the Anopheles gambiae heterochromatin
}

\author{
Maria V Sharakhova', Phillip George ${ }^{1}$, Irina V Brusentsova², Scotland C Leman ${ }^{3}$, Jeffrey A Bailey ${ }^{4}$, \\ Christopher D Smith ${ }^{5,6}$, Igor $\vee$ Sharakhov ${ }^{1^{*}}$
}

\begin{abstract}
Background: Heterochromatin plays an important role in chromosome function and gene regulation. Despite the availability of polytene chromosomes and genome sequence, the heterochromatin of the major malaria vector Anopheles gambiae has not been mapped and characterized.

Results: To determine the extent of heterochromatin within the An. gambiae genome, genes were physically mapped to the euchromatin-heterochromatin transition zone of polytene chromosomes. The study found that a minimum of 232 genes reside in $16.6 \mathrm{Mb}$ of mapped heterochromatin. Gene ontology analysis revealed that heterochromatin is enriched in genes with DNA-binding and regulatory activities. Immunostaining of the An. gambiae chromosomes with antibodies against Drosophila melanogaster heterochromatin protein 1 (HP1) and the nuclear envelope protein lamin $\mathrm{Dm}_{0}$ identified the major invariable sites of the proteins' localization in all regions of pericentric heterochromatin, diffuse intercalary heterochromatin, and euchromatic region $9 \mathrm{C}$ of the $2 \mathrm{R}$ arm, but not in the compact intercalary heterochromatin. To better understand the molecular differences among chromatin types, novel Bayesian statistical models were developed to analyze genome features. The study found that heterochromatin and euchromatin differ in gene density and the coverage of retroelements and segmental duplications. The pericentric heterochromatin had the highest coverage of retroelements and tandem repeats, while intercalary heterochromatin was enriched with segmental duplications. We also provide evidence that the diffuse intercalary heterochromatin has a higher coverage of DNA transposable elements, minisatellites, and satellites than does the compact intercalary heterochromatin. The investigation of $42-\mathrm{Mb}$ assembly of unmapped genomic scaffolds showed that it has molecular characteristics similar to cytologically mapped heterochromatin.

Conclusions: Our results demonstrate that Anopheles polytene chromosomes and whole-genome shotgun assembly render the mapping and characterization of a significant part of heterochromatic scaffolds a possibility. These results reveal the strong association between characteristics of the genome features and morphological types of chromatin. Initial analysis of the An. gambiae heterochromatin provides a framework for its functional characterization and comparative genomic analyses with other organisms.
\end{abstract}

\section{Background}

Located in pericentric, telomeric, and some internal chromosomal regions, heterochromatin plays an important role in cell division [1], meiotic pairing [2], regulation of DNA replication, and gene expression [3]. Among insect species, the most detailed analysis of heterochromatin has been performed in Drosophila [4-7]. Molecular analysis has determined that pericentric heterochromatic regions are enriched with highly and moderately repetitive DNA

\footnotetext{
* Correspondence: igor@vt.edu

'Department of Entomology, Virginia Tech, Blacksburg, VA 24061, USA

Full list of author information is available at the end of the article
}

sequences, and are extremely depleted of genes [8-10]. Mapping of heterochromatic scaffolds is difficult because the heterochromatin is underreplicated and poorly banded in polytene chromosomes of salivary glands. Special efforts had to be directed towards the assembly and annotation of heterochromatin in Drosophila [10-14]. Bioinformatic analysis of the heterochromatic portion of the Drosophila genome revealed the presence of more than 200 genes. Interestingly, heterochromatic genes are enriched specific functional domains, including putative membrane cation transporters domains and domains involved in DNA or protein binding [12]. This finding
C Biomed Central

(c) 2010 Sharakhova et al; licensee BioMed Central Ltd. This is an Open Access article distributed under the terms of the Creative Commons Attribution License (http://creativecommons.org/licenses/by/2.0), which permits unrestricted use, distribution, and reproduction in any medium, provided the original work is properly cited. 
suggests that pericentric heterochromatin may encode genes involved in the establishment or maintenance of alternative chromatin states. In addition to the pericentric heterochromatin, Drosophila has intercalary heterochromatin, which is interspersed throughout the euchromatin and characterized, in part, by underreplication in polytene chromosomes of larval salivary glands $[15,16]$. A study of a genome-wide profile of underreplication in polytene chromosomes identified 52 underreplication zones, which were colocalized with regions of intercalary heterochromatin. These underreplication zones varied from 100 to $600 \mathrm{~kb}$ in length, and each contained from 6 to 41 unique genes [17].

One of the important problems of chromosome biology is to understand the relationships between the morphology of the chromatin and the DNA and protein composition. Two morphological types of the heterochromatin have been described in the pericentromeric regions of Drosophila polytene chromosomes: proximal condensed, $\alpha$-, and distal diffuse, $\beta$-heterochromatin [18]. The compact central part of the chromocenter ( $\alpha$-type) is enriched with satellite DNA, while the distal diffuse area ( $\beta$-type) contains mostly transposable elements (TEs) $[19,20]$. Biochemical studies have discovered that heterochromatic regions have a specific histone code, characterized by hypoacetylation and methylation of the histone $\mathrm{H} 3$ at lysine 9 [21]. This modification of the histone $\mathrm{H} 3$ is a docking site for the heterochromatin protein 1 (HP1) [22,23], a major component of heterochromatin first described in Drosophila [24]. Comparative studies of Drosophila polytene chromosomes have discovered differences in the chromatin state suggesting the switching of chromatin states during evolution. For instance, when staining patterns of HP1 on polytene chromosomes were compared, it was found that the heterochromatic fourth chromosomes of $D$. melanogaster and D. pseudoobscura bind to HP1, while the euchromatic fourth chromosome of $D$. virilis does not. Interestingly, the level of CA/GT repeats on chromosome 4 of $D$. virilis is 20 fold higher than the level on chromosome 4 of $D$. melanogaster. Moreover, the density of TEs in this chromosome is significantly higher for D. melanogaster than for D. virilis [25-27].

A number of studies have demonstrated direct associations between heterochromatin and the nuclear envelope (NE) [28-33]. In Drosophila salivary gland nuclei, pericentromeric heterochromatin attaches permanently to the NE, while intercalary heterochromatin forms high-frequency contacts to NE [34]. Chromatin fibers of diffuse heterochromatin form visible attachments to the NE in Drosophila [30] and Anopheles $[35,36]$. The chromosomal regions that attach to the NE may depend on the presence of specific DNA. For example, repetitive matrix attachment regions (MARs) specifically bind to lamin, the major protein of the nuclear periphery [28,37-40]. It has been shown that MAR DNA is several fold richer in heterochromatin than in euchromatin [41-43].

Although the Drosophila studies provided important insights into the structural and functional organization of heterochromatin, the organization of heterochromatin in other insects remains poorly understood. Malaria mosquitoes are an excellent system for studying heterochromatin because they possess well-developed polytene chromosomes with clear morphology. Sequencing of the genome of the major African malaria vector An. gambiae [44] provides an opportunity to analyze the molecular structure of the heterochromatin and to study genomic determinants of heterochromatin formation, maintenance, and function. In malaria mosquitoes, the heterochromatin size and morphology vary significantly among species and within species [45-47], affecting mating behavior and fertility $[48,49]$. In the $A n$. gambiae complex, one of the species, An. gambiae sensu stricto, is subdivided into two subtaxa: the $\mathrm{M}$ and $\mathrm{S}$ molecular forms [50]. These two partially isolated subtaxa predominantly breed within their own form and differ in behavior and environmental adaptations [51]. A DNA microarray analysis revealed that two pericentric regions on $\mathrm{X}$ and $2 \mathrm{~L}$ were the major islands of fixed genomic differentiation between the $M$ and S molecular forms [52]. A more recent microarray study based on the improved AgamP3 assembly and AgamP3.4 gene build provided better estimates for the number and size of diverged pericentric islands between the $\mathrm{M}$ and $\mathrm{S}$ forms [53]. The study found three islands of genomic divergence: $\mathrm{a} \sim 4-\mathrm{Mb}$ region on the $\mathrm{X}$ chromosome, a $\sim 2.5-\mathrm{Mb}$ region on the $2 \mathrm{~L}$ arm, and a $1.7-\mathrm{Mb}$ region on the $3 \mathrm{~L}$ arm. However, it is not clear if the pericentric islands of genomic divergence are located within heterochromatin or mostly overlap with euchromatin of An. gambiae.

According to the $\mathrm{C}_{\mathrm{o}} \mathrm{T}$ analysis, about $86 \mathrm{Mb}$ (33\% of 260-Mb genome) of the $A n$. gambiae genome corresponds to repetitive elements, which are mostly located in heterochromatic areas of the chromosomes [54]. However, only $3.3 \mathrm{Mb}$ were identified as heterochromatin in the first publication of An. gambiae genome [44]. Using cDNA clones for the physical mapping of the heterochromatic scaffolds, an additional 5.3 $\mathrm{Mb}$ were mapped to the pericentromeric regions in the chromosomes [55]. Nevertheless, the more precise chromosomal and genomic mapping, as well as detailed analysis of the molecular organization of the Anopheles heterochromatin, has yet to be conducted.

In this study, the boundaries of the heterochromatineuchromatin junctions of all morphologically defined pericentric and intercalary heterochromatin regions were determined for each of the five chromosomal arms 
of An. gambiae. The large regions of intercalary heterochromatin were morphologically different: $0.7-\mathrm{Mb}$ and $0.8-\mathrm{Mb}$ regions of $2 \mathrm{~L}$ and $3 \mathrm{~L}$ were diffuse, while a 2.9-Mb region of $3 \mathrm{R}$ was a compact heterochromatin. Because the $A n$. gambiae genome assembly successfully captured not only the euchromatin, but a significant portion of the heterochromatin, comparative analysis of chromatin types was possible. We provided evidence that heterochromatin and euchromatin differ in gene density and the coverage of retroelements and segmental duplications (SDs). Gene ontology (GO) analysis revealed that heterochromatin is enriched in genes with DNA-binding and regulatory activities. The pericentric heterochromatin had the highest coverage of retroelements and tandem repeats, while intercalary heterochromatin was enriched with SDs. We also demonstrated that the diffuse intercalary heterochromatin binds to HP1 and lamin and has a higher coverage of DNA TEs, minisatellites, and satellites than does the compact intercalary heterochromatin. The investigation of $42-\mathrm{Mb}$ assembly of unmapped genomic scaffolds ("unknown chromosome") demonstrated that it has molecular characteristics similar to cytologically mapped heterochromatin. Finally, the locations and sizes of pericentric heterochromatin regions closely matched the locations and sizes of pericentric islands of genomic divergence between $\mathrm{M}$ and $\mathrm{S}$ incipient species of $A n$. gambiae.

\section{Results and Discussion}

Morphological types of the An. gambiae heterochromatin The diploid number of the chromosomes in malaria mosquitoes is six, which includes two pairs of autosomes as well as the $\mathrm{X}$ and $\mathrm{Y}$ sex chromosomes. The polytene chromosome complement of a female mosquito has five chromosomal arms: four autosomal arms 2R, 2L, 3R, 3L, and one arm of the $\mathrm{X}$ chromosome. In this study, morphological identification of the heterochromatin for the African malaria mosquito An. gambiae was performed for the first time. The following criteria were used to distinguish heterochromatic and euchromatic regions in the polytene chromosomes from ovarian nurse cells (Figure 1). We considered a region as heterochromatic if it (i) consisted of a compact condensed block or (ii) had a diffuse granulated structure with no banding pattern. These two types of heterochromatin can be distinguished from euchromatic regions, which have a clear banding pattern or puffy nongranulated areas. Pericentric regions of all chromosomes matched these morphological criteria of heterochromatin. The pericentric heterochromatin of the $\mathrm{X}$ chromosome has a large diffuse granulated area in region 6 , which is similar to the $\beta$-heterochromatin of Drosophila (Figure 2a). The diffuse granulated heterochromatin (Figure 2a) is morphologically distinct from the euchromatic nongranulated puff in subdivision $9 \mathrm{C}$ of the $2 \mathrm{R}$ arm (Figure $2 \mathrm{~b}$ ). In addition, region 6 of the $\mathrm{X}$ chromosome has a dark compact band in the tip of the chromosome (Figure 1), which was previously described as a nucleolar organizer region because ribosomal genes were mapped to this area by in situ hybridization [55]. The polytene chromosome 2 has a dark compact proximal heterochromatin surrounded by abundant diffuse heterochromatin in regions 19E-20A (Figure 2c). A dark heterochromatic band is also present in region 19D of the $2 \mathrm{R}$ arm. The pericentric heterochromatin of chromosome 3 spans subdivisions 37D-38A. Chromosomes 2 and 3 form a diffuse chromocenter via their pericentric heterochromatin [36].

Three regions of intercalary heterochromatin are visible on arms 2L, 3R, and 3L (Figure 2c). The subdivision 21A of $2 \mathrm{~L}$ chromosomal arm forms a large, lightly granulated puff-like structure with no banding pattern. The middle area of subdivision $38 \mathrm{C}$ of $3 \mathrm{~L}$ arm has a similar morphology, but it is slightly smaller and darker. Both regions of intercalary diffuse heterochromatin are located in close proximity to the pericentric regions. The third region of intercalary heterochromatin is in subdivision $35 \mathrm{~B}$ of the $3 \mathrm{R}$ arm and is located 10 subdivisions away from the centromere. Unlike intercalary heterochromatin of $2 \mathrm{~L}$ and $3 \mathrm{~L}$, this region has a compact dense structure, which is similar to $\alpha$-heterochromatin of Drosophila. In malaria mosquitoes, diffuse and compact types of heterochromatin were previously described in the Anopheles maculipennis subgroup [35,56]. Interestingly, the large blocks of compact heterochromatin or the diffuse intercalary heterochromatin regions have not been seen in most species of Drosophila. The intercalary heterochromatin in salivary gland nuclei of D. melanogaster is strongly underreplicated and has the morphology of "weak" points, which are able to form ectopic contacts [57]. These properties are less prominent in ovarian nurse cell nuclei of the $D$. melanogaster otu ${ }^{11}$ strain where the bands of intercalary heterochromatin are morphologically similar to euchromatic bands [58]. Large blocks of intercalary heterochromatin have been described in polytene chromosomes of $D$. immertensis and species from genera Chironomus and Anopheles $[4,56]$. Although the morphology of pericentric heterochromatin is similar in An. gambiae and D. melanogaster, the presence of two distinct types of intercalary heterchromatin in An. gambiae makes this species a unique model system for studying genomic determinants of chromatin morphology.

\section{Chromosomal localization of HP1 and lamin in An. gambiae}

HP1 is an evolutionarily conserved protein and a good marker of heterochromatic regions [25]. One HP1a ortholog is present in An. gambiae (VectorBase gene ID: 


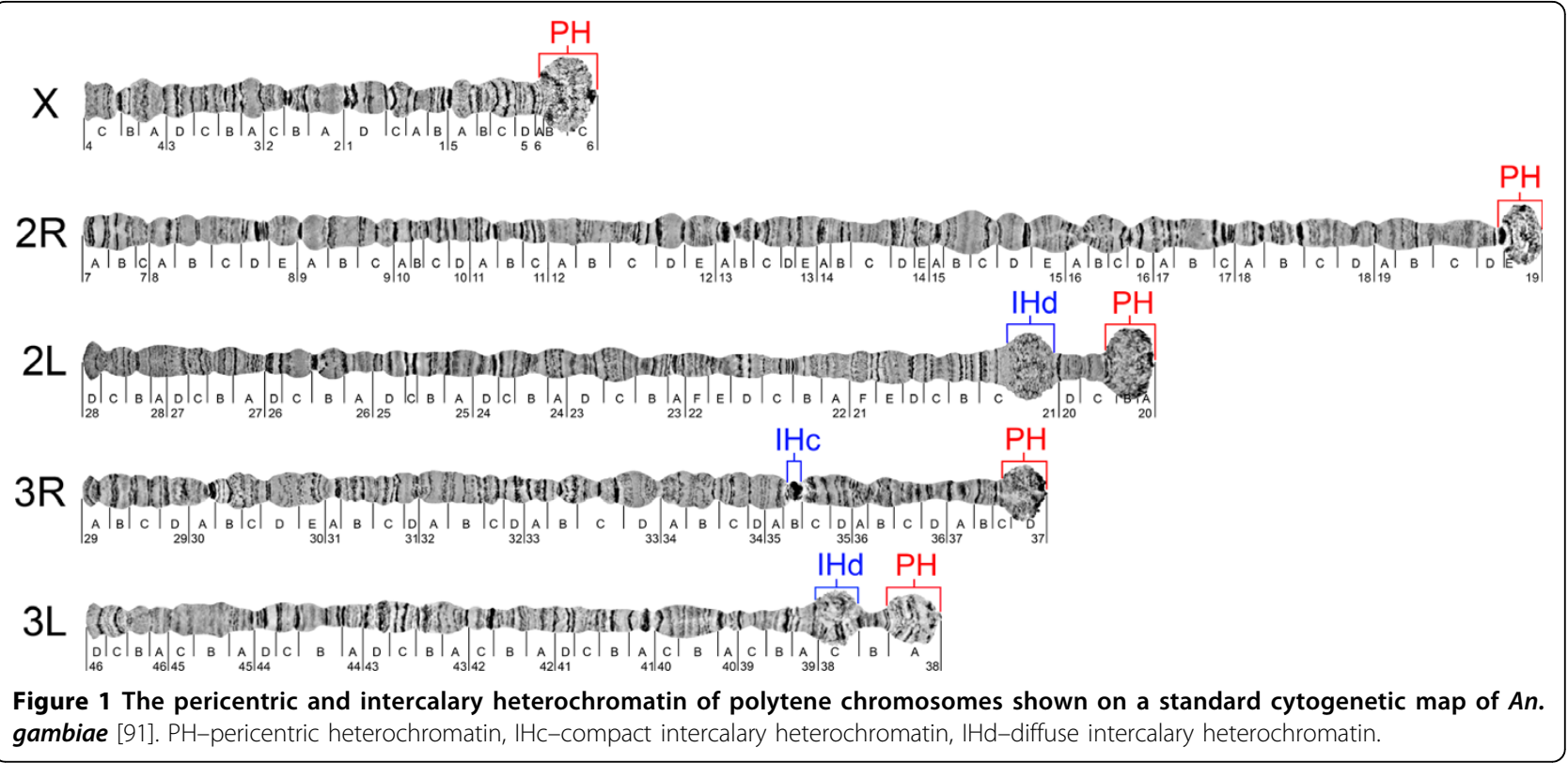

AGAP009444). The An. gambiae protein AGAP009444PA is $70.4 \%$ similar to the D. melanogaster HP1a protein in the 206 overlapping amino acids. The antibodies for HP1 were localized in the chromocenter, chromosome 4 , telomeric, and some euchromatic regions in D. melanogaster [24,59]. In order to examine the association of HP1 with heterochromatin in An. gambiae, we hybridized the primary antibody C1A9 against D. melanogaster HP1 to An. gambiae polytene chromosomes. This antibody correctly recognized HP1 even in more distantly related species such as the mealybug Planococcus citri [60]. Several positively stained loci were invariable, i.e., they were found on every examined chromosome and on every slide. Similar to Drosophila, the major invariable sites of HP1 localization were the pericentric regions in $A n$. gambiae (Figure 2). In addition, diffuse intercalary heterochromatin of regions $21 \mathrm{~A}$ and $38 \mathrm{C}$ were always stained positively for HP1. Only one major invariable HP1-binding site was identified in a large interband of the euchromatic region $9 \mathrm{C}$ of $2 \mathrm{R}$ arm (Figure 2b). All other positive euchromatic sites were variable, and a total of $122 \mathrm{HP} 1$ binding sites were detected on An. gambiae chromosomes (Table 1). Based on the previous $A n$. gambiae genome mapping coordinates, we analyzed the molecular content of the euchromatic site of HP1/lamin binding in region 9C (genome coordinates 12874430-13778780). The analysis found no enrichment of any class of TE. The only heterochromatic molecular feature of this region was a $4.5-\mathrm{kb}$ block of satellite DNA, which consisted of 228-bp units repeated 40 times. Similarly, one major invariable site of HP1 binding was found in euchromatic region 31 of the 2L arm in D. melanogaster [61]. However, the molecular analysis of this region found no enrichment in any repetitive DNA. About 200-300 actively expressing loci related to developmentally important and heat-shock genes were positively stained for HP1 in Drosophila chromosomes, suggesting a positive role for HP1 in euchromatic gene expression [62-64]. However, only 20 HP1-positive euchromatic sites were invariable among strains, natural populations, and individuals of D. melanogaster [61]. Unlike in Drosophila, telomeric localization of HP1 was found only on chromosome X in $A n$. gambiae, but even this site was variable. Surprisingly, no HP1 binding was detected in the compact intercalary heterochromatin of subdivision $35 \mathrm{~B}$ of the $3 \mathrm{R}$ chromosome, suggesting that this region has a distinct molecular composition or is strongly underreplicated, and thus, HP1 presence is below the level of detection. Subdivision 35B was morphologically described as heterochromatic based on very dense dark structure (Figure 1 and 2c). The genomic analysis confirmed its repeat-rich gene-poor heterochromatic nature (see "Difference in molecular content among chromatin types of An. gambiae").

Association of heterochromatin with the NE has been demonstrated in a number of studies [28-33]. Attachment of pericentric regions to the NE in ovarian nurse cell nuclei of An. gambiae has also been demonstrated [36]. In our study, the attachments to the nuclear periphery were detected in all pericentric regions, and diffuse intercalary heterochromatin in regions $21 \mathrm{~A}(2 \mathrm{~L})$ and $38 \mathrm{C}(3 \mathrm{~L})$ (Figure 2d). To test whether heterochromatin binds to the NE, mosquito chromosomes were stained with antibody ADL67.10 against NE protein lamin $\mathrm{Dm}_{0}$ of $D$. melanogaster. We found only one 


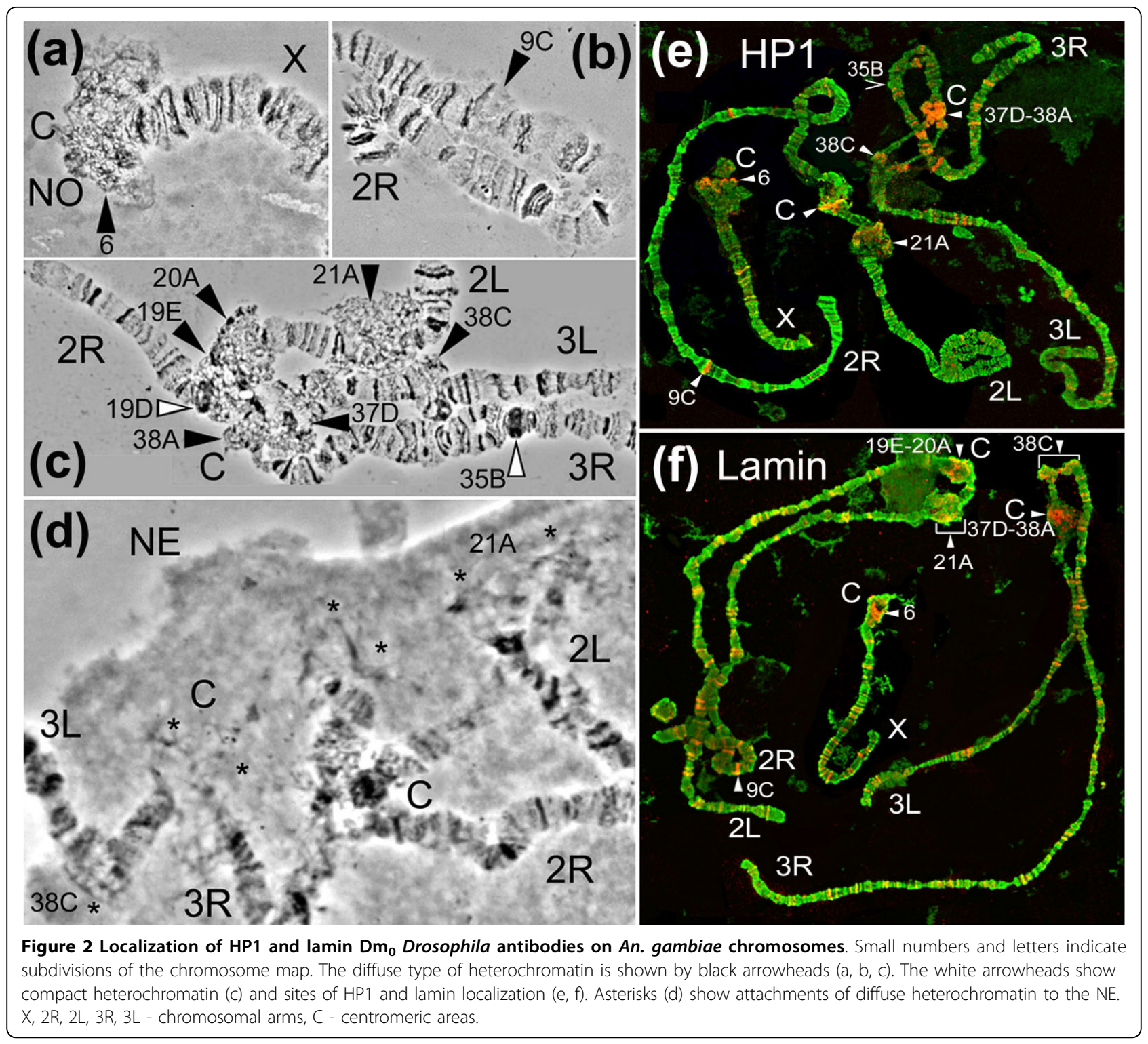

Table 1 Localization of HP1 and lamin on An. gambiae chromosomes

\begin{tabular}{|c|c|c|c|c|}
\hline Chromosome & Invariable sites & Variable sites & Average number of variable sites per $\mathrm{Mb}$ & Chromosome analyzed \\
\hline $\mathrm{X:HP1}$ & 1 & $1-17$ & 0.33 & 10 \\
\hline X: Lamin & 1 & $1-19$ & 0.41 & 5 \\
\hline 2R: HP1 & 2 & $13-53$ & 0.55 & 3 \\
\hline 2R: Lamin & 2 & $7-49$ & 0.46 & 5 \\
\hline 2L: HP1 & 2 & $2-20$ & 0.22 & 4 \\
\hline 2L: Lamin & 2 & $17-31$ & 0.49 & 5 \\
\hline 3R: HP1 & 1 & $1-18$ & 0.18 & 3 \\
\hline 3R: Lamin & 1 & 29 & 0.55 & 2 \\
\hline 3L: HP1 & 2 & $2-14$ & 0.19 & 2 \\
\hline 3L: Lamin & 2 & 30 & 0.71 & 1 \\
\hline
\end{tabular}


lamin Dm0 ortholog in the An. gambiae genome (VectorBase gene ID: AGAP011938). The An. gambiae protein AGAP011938-PA is $78.2 \%$ similar to the $D$. melanogaster lamin $\mathrm{Dm}_{0}$ protein in the 628 overlapping amino acids. The antibody against lamin $\mathrm{Dm}_{0}$ successfully hybridized to the An. gambiae chromosomes and colocalized with the HP1 antibody in all major invariable sites and in most of the variable sites (Figure $2 \mathrm{f}$ ). However, the total number of sites was higher for lamin $\mathrm{Dm}_{0}$ (158 sites) than for HP1 (122 sites) (Table 1). The major sites for lamin $\mathrm{Dm}_{0}$ were found in the pericentromeric areas, diffuse intercalary heterochromatin regions, and euchromatic interband in region $9 \mathrm{C}$. No lamin $\mathrm{Dm}_{0}$ antibody was detected in region $35 \mathrm{~B}$ of the $3 \mathrm{R}$ chromosome of An. gambiae.

Thus, the immunostaining of the antibodies for HP1 and lamin $\mathrm{Dm}_{0}$ has demonstrated that both proteins are primarily associated with the diffuse pericentric and intercalary heterochromatin, but not with the compact intercalary heterochromatin of An. gambiae. Two binding motifs, chromo and chromoshadow domains, provide HP1 with the ability to be broadly involved in chromatin and protein binding [65-67]. In vitro studies revealed a direct interaction between HP1 and the lamin $B$ receptor in mammalian cells $[33,68,69]$. However, in Drosophila, similar direct associations of HP1 with lamin have not been shown, and these proteins have been found associated with different genomic regions [70]. Therefore, despite the colocalization of HP1 and lamin in heterochromatin of An. gambiae, the actual protein binding sites in the genome may differ as suggested by the additional regions of lamin binding.

\section{Heterochromatin-euchromatin boundaries in the An. gambiae genome}

The cytological identification of heterochromatin allowed us to determine the location of heterochromatin-euchromatin boundaries in the An. gambiae genome. The approximate coordinates were found based on the genome positions of BAC and cDNA clones, which were physically mapped to chromosomes near heterochromatin-euchromatin boundaries $[44,55]$. Because heterochromatic regions were not sufficiently covered with markers, additional PCR-amplified gene fragments were designed and utilized as DNA probes for physical mapping. Fluorescent in situ hybridization (FISH) was used to hybridize multiple PCR products thought to be located near the heterochromatin-euchromatin boundary of each major heterochromatic region of the five chromosome arms (Table 2). This allowed for more exacting definition of the boundaries, based on the outermost heterochromatin and euchromatin markers, defining a transition zone with an average size of $78 \mathrm{~kb}$ (range: $\sim 15$ to $226 \mathrm{~kb}$ ). Based on these boundaries, a total of $\sim 16.6 \mathrm{Mb}$ was defined as a heterochromatin in the currently mapped genome assembly of $A n$. gambiae (Figure 3a). The mapped portion of the heterochromatin within defined chromosomes now comprises $\sim 6.4 \%$ of the $\sim 260-\mathrm{Mb}$ genome $[44,54]$ and contains $232(\sim 1.8 \%)$ of the $\sim 13,000$ total predicted genes. For comparison, no less than 230 genes were annotated in $24 \mathrm{Mb}$ of D. melanogaster heterochromatin (release 5.1) [12]. In addition, the sizes of intercalary heterochromatin were also determined. The diffuse heterochromatic regions were $0.7 \mathrm{Mb}$ and $0.8 \mathrm{Mb}$ in $2 \mathrm{~L}$ and $3 \mathrm{~L}$, respectively, and the compact heterochromatin on $3 \mathrm{R}$ was $2.9 \mathrm{Mb}$ long. The relatively short sizes of regions of intercalary diffuse heterochromatin as compared to regions of condensed heterochromatin suggest incomplete genome assembly of the diffuse type. However, these sizes exceed the sizes of intercalary heterochromatin known in Drosophila, which range from 100 to $600 \mathrm{~kb}$ [17]. The higher repeat content of the mosquito genome may be responsible for the larger sizes of intercalary heterochromatin in An. gambiae.

\section{Heterochromatin and pericentric regions of genomic divergence in incipient species}

Three pericentric islands of genomic divergence were found in chromosomes $\mathrm{X}, 2 \mathrm{~L}$, and $3 \mathrm{~L}$ in two partially isolated subtaxa - the $\mathrm{M}$ and $\mathrm{S}$ molecular forms of An. gambiae s.s. [53]. Our analysis showed that the positions of islands of genomic divergence mostly correspond to the positions of physically mapped regions of pericentric heterochromatin (Figure $3 \mathrm{~b}$ ). The sizes of the pericentric heterochromatin were the following: 4.4 $\mathrm{Mb}$ of the $\mathrm{X}$ chromosome, 2.4 $\mathrm{Mb}$ of the $2 \mathrm{~L}$ arm, and $1.8 \mathrm{Mb}$ of the $3 \mathrm{~L}$ arm. Thus, the overlaps with islands of genomic divergence are $91 \%$ in the $\mathrm{X}$ chromosome, $97 \%$ in the $2 \mathrm{~L}$ arm, and $94 \%$ in the $3 \mathrm{~L}$ arm. This observation suggests that heterochromatic sequences diverge rapidly during speciation of malaria mosquitoes. Earlier cytological studies showed the presence of significant intra- and interspecific differences in amount and location of heterochromatin in the An. gambiae complex $[45,48]$. A genome-wide microsatellite study of members of the An. gambiae complex has determined a high level of genetic introgression among species [71]. However, the An. gambiae microsatellites at six loci of $\mathrm{X}, 3 \mathrm{~L}$, and $3 \mathrm{R}$ could not be amplified in all sibling species, indicating significant sequence divergence from the major malaria vector. These loci were identified as heterochromatic in our study. Fast changes in heterochromatic DNA can be accompanied by the rapid evolution of heterochromatic proteins. Although HP1 is an evolutionarily conserved protein, other heterochromatin- and centromere-associated proteins demonstrate rapid adaptive evolution [72,73]. For example, an LHR protein 
Table 2 Boundaries between heterochromatin and euchromatin in the An. gambiae genome

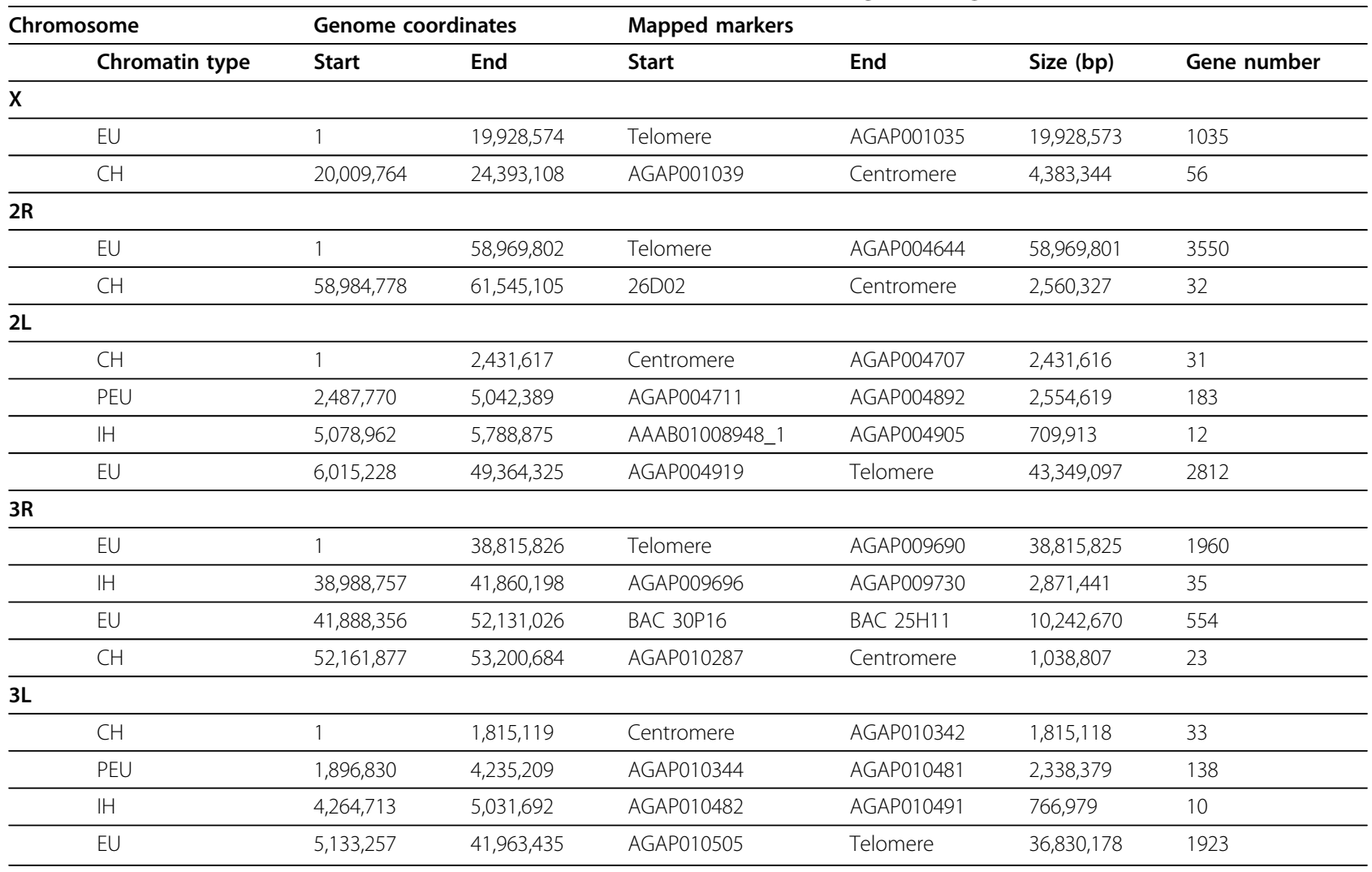

encoded by $l h r$ (Lethal hybrid rescue) colocalizes with HP1 in heterochromatic regions and has diverged extensively in sequence between $D$. melanogaster and $D$. simulans species in a manner consistent with positive selection. Interestingly, F1 hybrids between these species demonstrate altered chromatin structure, probably attributable to the effects of species-specific differences in
TEs and other repetitive DNAs [74], suggesting a role for heterochromatin in speciation.

\section{Overrepresentation of gene ontology terms in the}

An. gambiae heterochromatin

To characterize gene content of the An. gambiae heterochromatin, we utilized GO terms [75]. The frequencies

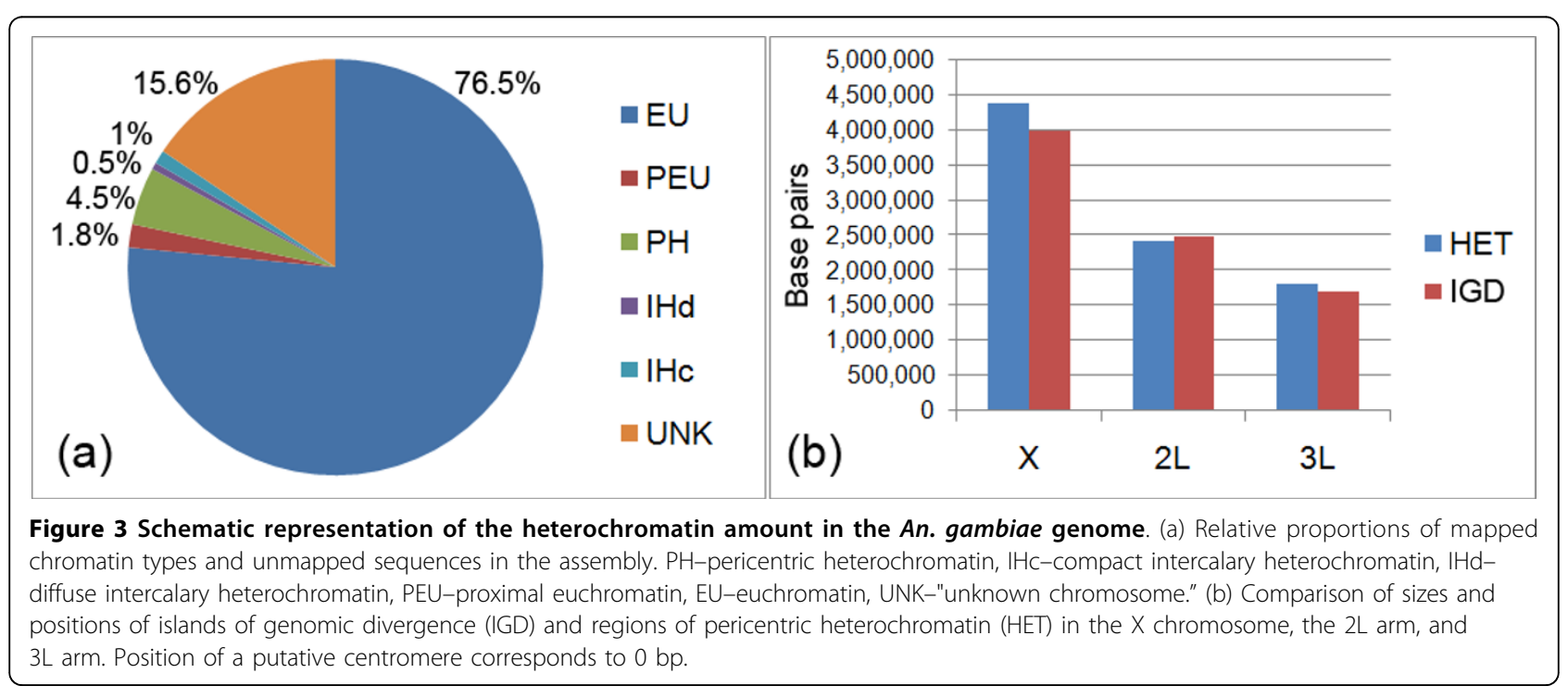




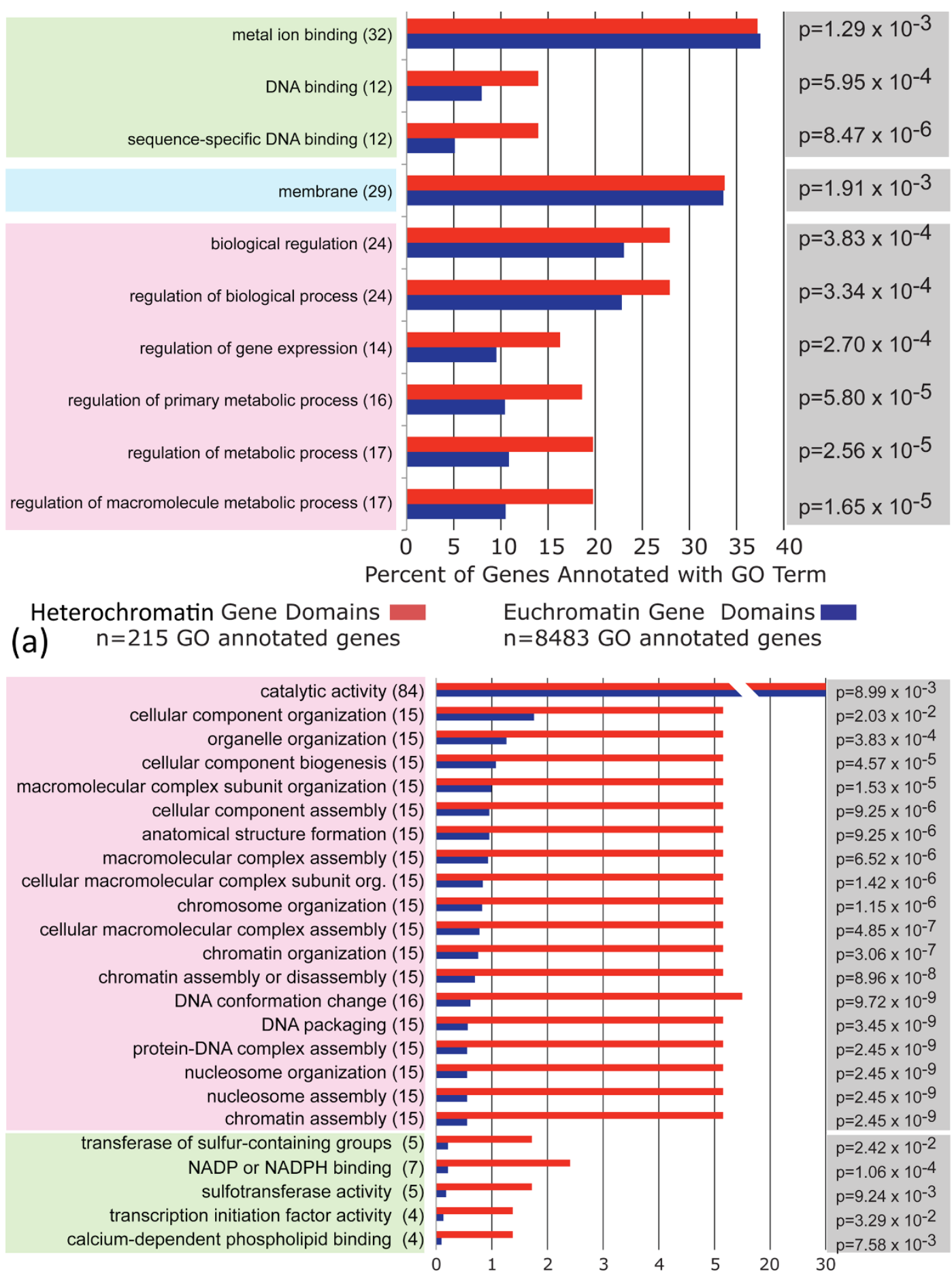

Percent of Genes Annotated with GO Term

\section{(b) Unmapped Gene Domains}

\section{Euchromatin Gene Domains $\mathrm{n}=8483 \mathrm{GO}$ annotated genes}

Figure 4 Overrepresented GO terms in genes within the cytologically confirmed heterochromatin (a) and within "unknown chromosome" (b) of An. gambiae. The percentages of heterochromatic (red) and euchromatic (blue) genes containing the listed GO biological process (pink shading), cellular location (blue shading), and molecular function (green shading) terms are indicated. Numbers in parentheses refer to the actual number of heterochromatin or unmapped genes annotated with the listed GO domain. GO-Term-Finder, Bonferroni corrected p-value scores are shown to the right (grey shading). 
of GO terms assigned to genes in heterochromatin were compared to frequencies for all GO-annotated genes in the peptide dataset of An. gambiae (Figure 4a). After Bonferroni correction for multiple tests, this analysis revealed significant enrichment for molecular functions in heterochromatin, including DNA binding (12 genes) and sequence-specific DNA binding (12 genes). Protein products of 29 heterochromatic genes constitute membrane, representing a significant enrichment of the GO cellular location. Finally, heterochromatin had overrepresentation of several gene types, including those encoding for proteins involved in biological regulation (24 genes) and regulation of metabolic processes (17 genes) (biological processes). The GO analysis of the "unknown chromosome" (sequence assembly lacking chromosomal assignment) identified enrichment in a number of interesting genes (Figure $4 \mathrm{~b}$ ). We found that genes residing in the "unknown chromosome" had significant overrepresentation of GO terms in biological processes, including chromosome organization (15 genes), DNA packaging (15 genes), and nucleosome assembly (15 genes). Transcription initiation factor activity (four genes) was among several molecular functions overrepresented in the genes within the "unknown chromosome." Analysis of the heterochromatic portion of the Drosophila genome revealed the overrepresentation of similar GO terms [12]. These studies suggest that heterochromatin of insects may accumulate genes important for its own establishing, maintaining, or modifying chromatin structure.

\section{Difference in molecular content among chromatin types of An. gambiae}

Using Bayesian statistical model and procedure for discerning differences between chromatin types, eight molecular features were analyzed: genes, DNA-mediated TEs (DNA TEs), RNA-mediated TEs (RNA TEs), SDs, micro- and minisatellites, satellites, and MARs. These molecular features were compared among five distinct chromatin types: 1 ) pericentric heterochromatin of all chromosomes; 2) diffuse intercalary heterochromatin in regions $21 \mathrm{~A}$ of $2 \mathrm{~L}$ and $38 \mathrm{C}$ of $3 \mathrm{~L} ; 3$ ) compact intercalary heterochromatin, region $35 \mathrm{~B}$ of $3 \mathrm{R}$; 4) proximal euchromatin, located between pericentric and diffuse intercalary heterochromatin, includes subdivisions $20 \mathrm{CD}$ of $2 \mathrm{~L}$ and $38 \mathrm{~B}$ of $3 \mathrm{~L}$; and 5 ) euchromatin in all remaining regions in the chromosomes. For this analysis, the data that distinguishes both the counts and the overall basepair coverage were incorporated for each molecular feature into the genomic windows of each of the five chromatin types. Dominant model selection procedures gave us the ability to compare all possible competing models and to select between parsimonious models by maximizing the posterior distribution.
Heterochromatin had a uniformly low concentration of genes. On average, the gene density was 4.7 times lower in the heterochromatin than in the euchromatin (Additional file 1, Table S1). Our analysis showed that heterochromatin significantly exceeds euchromatin in the coverage of RNA TEs and SDs. RNA TEs were the most abundant features in the mosquito genome (Figure $5)$. The pericentric heterochromatin had the highest coverage of RNA TEs, microsatellites, minisatellites, and satellites. The intercalary heterochromatin had a higher coverage of SDs than all other chromatin types. The diffuse intercalary heterochromatin had a higher coverage of TEs, minisatellites, and satellites than did the compact intercalary heterochromatin. The enrichment of TEs in the pericentric heterochromatin and diffuse intercalary heterochromatin as compared to the compact intercalary heterochromatin can explain the pattern of HP1 localization in polytene chromosomes of An. gambiae. Pericentric and diffuse intercalary heterochromatin, but not the compact type, was HP1 positive. Similarly, the fourth chromosomes of D. melanogaster and D. pseudoobscura bound to HP1, while the fourth chromosome of $D$. virilis did not. The density of TEs in this chromosome was significantly higher for D. melanogaster than for D. virilis [25-27]. The proximal euchromatin had a higher coverage of DNA TEs, MARs, and SDs but a lower coverage of satellites than the rest of the euchromatin. These differences can probably be explained by the close distance of the proximal euchromatin to the centromere.

\section{Chromatin types and genome landscape in An. gambiae}

In addition to the overall differences among chromatin types, the distribution of molecular features within chromosomal arms was analyzed. A high density of genes was seen outside of the heterochromatin boundaries believed to be euchromatin, followed by a transition zone and a heterochromatic region with a low gene density. The distribution of TEs densities had the opposite pattern. The highest coverage of SDs was detected in intercalary heterochromatin with peaks in some euchromatic regions of the $2 \mathrm{R}, 3 \mathrm{R}$, and $3 \mathrm{~L}$ arms (Figure 6). MARs were found concentrated in the pericentric heterochromatic and proximal euchromatic regions of all arms, but they were also abundant in distal euchromatic regions of the $2 \mathrm{~L}$, $3 \mathrm{R}$, and $3 \mathrm{~L}$ arms. We observed the high coverage of predicted MARs in heterochromatic regions, which are associated with the NE [41-43]. Moreover, the increase in MAR coverage seen in euchromatic regions of the $2 \mathrm{~L}$, $3 \mathrm{R}$, and $3 \mathrm{~L}$ arms correlated positively with the higher density of lamin-positive sites in these arms detected by immunostaining (Table 1). The highest coverage of MARs was found in proximal euchromatin, which was not stained by the lamin antibody. Also, the two types of 


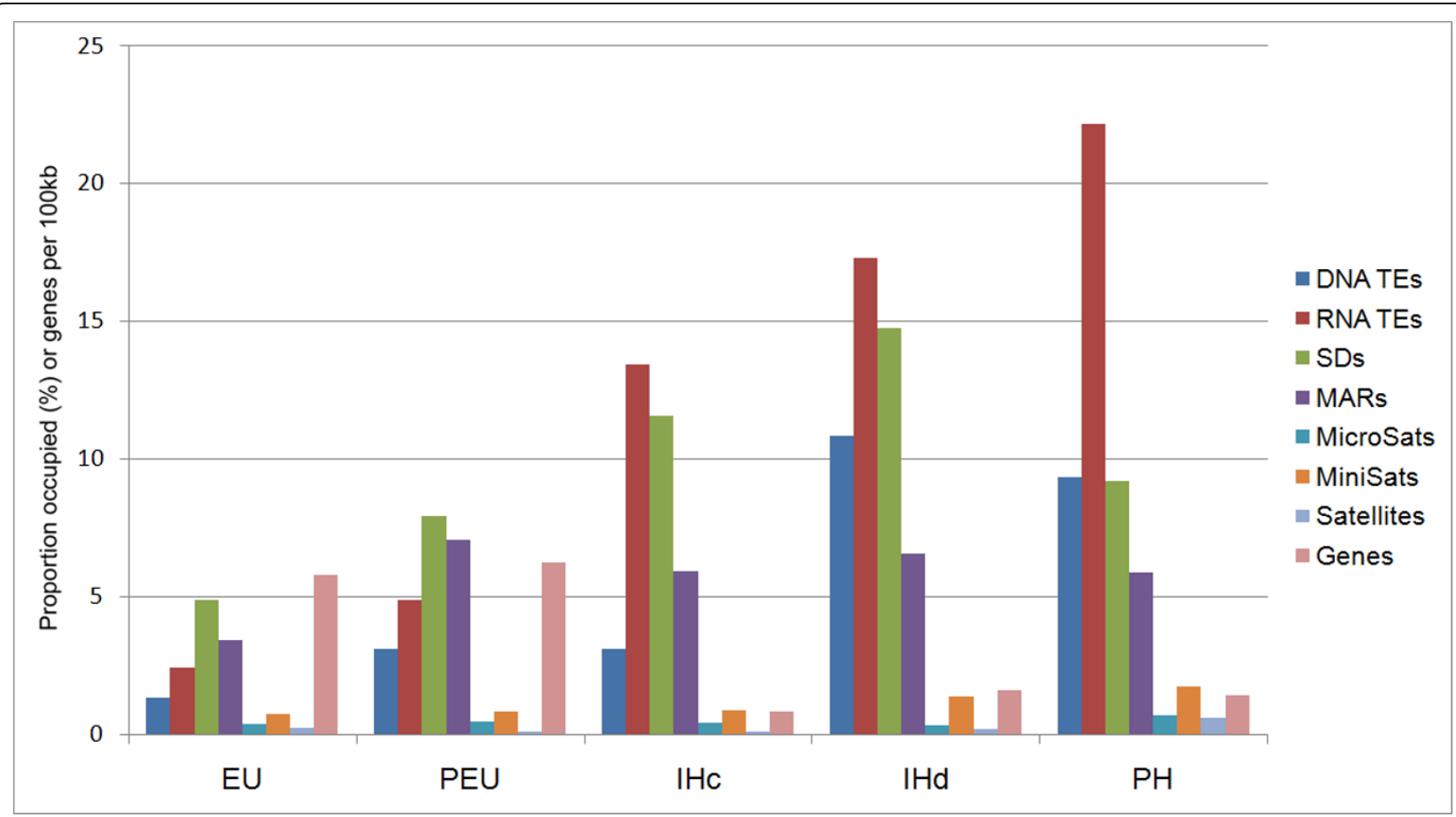

Figure 5 Median values of gene density and repetitive element coverage in chromatin types of An. gambiae. Percentage of region length occupied per $1 \mathrm{Mb}$ are indicated for all repetitive elements. PH-pericentric heterochromatin, $\mathrm{IHC}$-compact intercalary heterochromatin, IHd-diffuse intercalary heterochromatin, PEU-proximal euchromatin, EU-euchromatin.

heterochromatin were not significantly different in MAR coverage. However, the lamin-positive pericentric and diffuse intercalary heterochromatic regions were significantly enriched with TEs. The coverage of DNA TEs was about two times higher in pericentric and diffuse intercalary heterochromatin than in other chromatin types (Table S1).

Overall, this analysis confirmed morphological predictions of heterochromatin. All types of heterochromatin in the An. gambiae genome had typical heterochromatic molecular features: low gene density and high coverage of TEs and SDs. However, because TEs are significantly underannotated in the An. gambiae genome, meaningful comparisons of the TE content of heterochromatin between mosquito and fruit fly are difficult.

\section{Unmapped genome assembly of An. gambiae}

The unmapped portion of the AgamP3 An. gambiae genome assembly comprises $42 \mathrm{Mb}$ [76], i.e. $16 \%$ of the genome, and has 491 protein coding genes (Figure 3a). The analysis of the genomic content of this "unknown chromosome" (http://www.vectorbase.org/) revealed that the density of genes and the coverage of TEs and microsatellites were similar to that of the heterochromatin (Figure 7). The highest coverage of minisatellites and satellites was detected in the "unknown chromosome" suggesting that the majority of these scaffolds belong to heterochromatin. Two satellites, AgY477 and Ag53C, were mapped to the most proximal heterochromatin of the An. gambiae polytene chromosomes [77]. The location of satellite DNA in the proximal pericentric heterochromatin has also been demonstrated in An. stephensi [78]. An enrichment with highly repetitive DNA has been found in the compact heterochromatin of the $A n$. macullipennis subgroup [56]. Telomeres of the An. gambiae chromosomes do not display heterochromatic morphology. Subtelomeric regions possess typical euchromatic banding patterns. However, molecular analysis of the telomeric end of the $2 \mathrm{~L}$ arm demonstrated the presence of satellites and minisatellites $[79,80]$. Therefore, the unmapped portion of the An. gambiae genome assembly likely contains sequences from the most proximal pericentric, most distal telomeric ends of chromosomes, and intercalary diffuse heterochromatin. In D. melanogaster, $10 \mathrm{Mb}$ of the unmapped portion of the genome was also enriched in tandem repeats and satellites [12].

\section{Conclusion}

Morphological identification and detailed physical mapping allowed us to define an expanded compartment of recognizable heterochromatin with distinct molecular features within the An. gambiae genome assembly. Now about 16.6 Mb of mapped heterochromatin with 


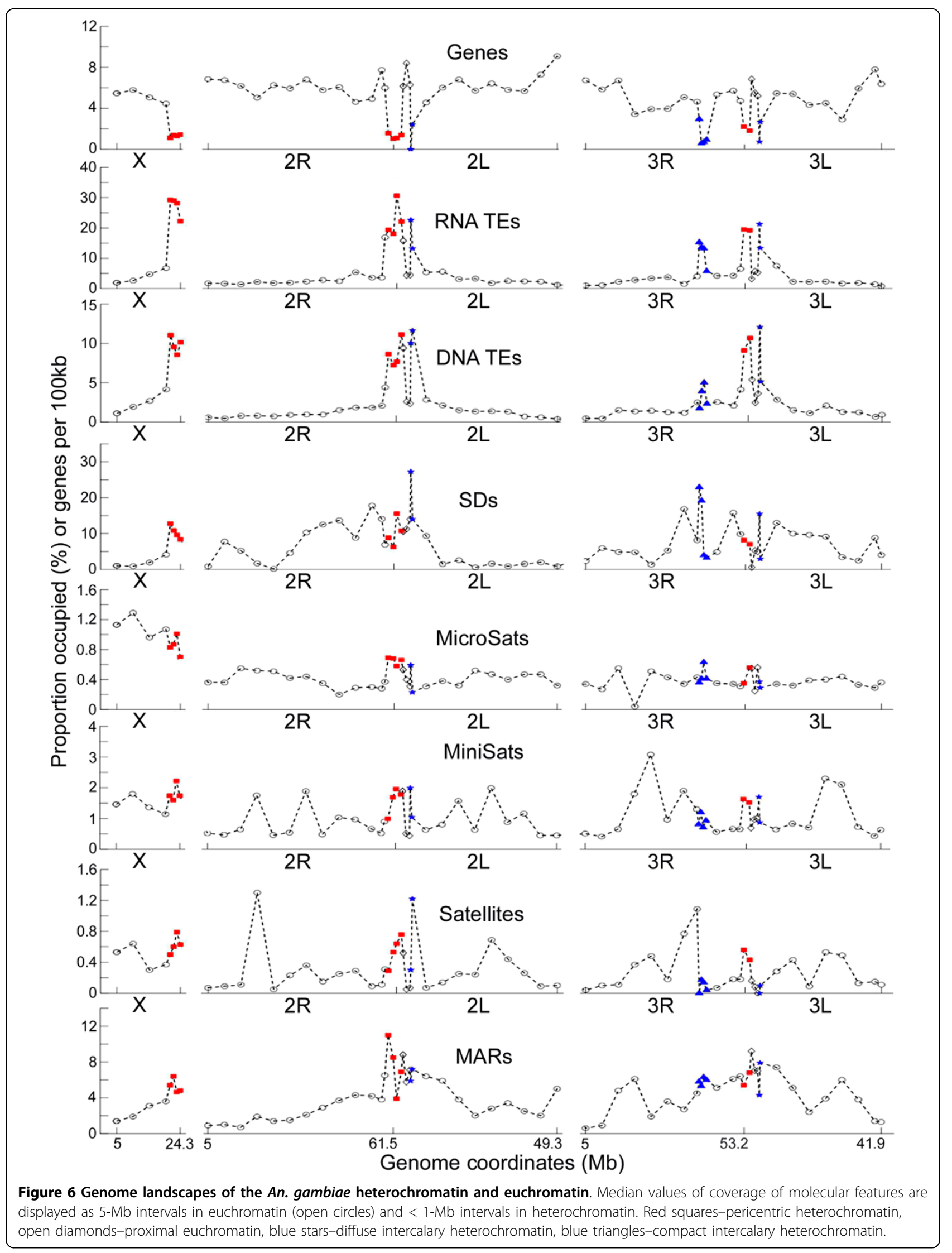




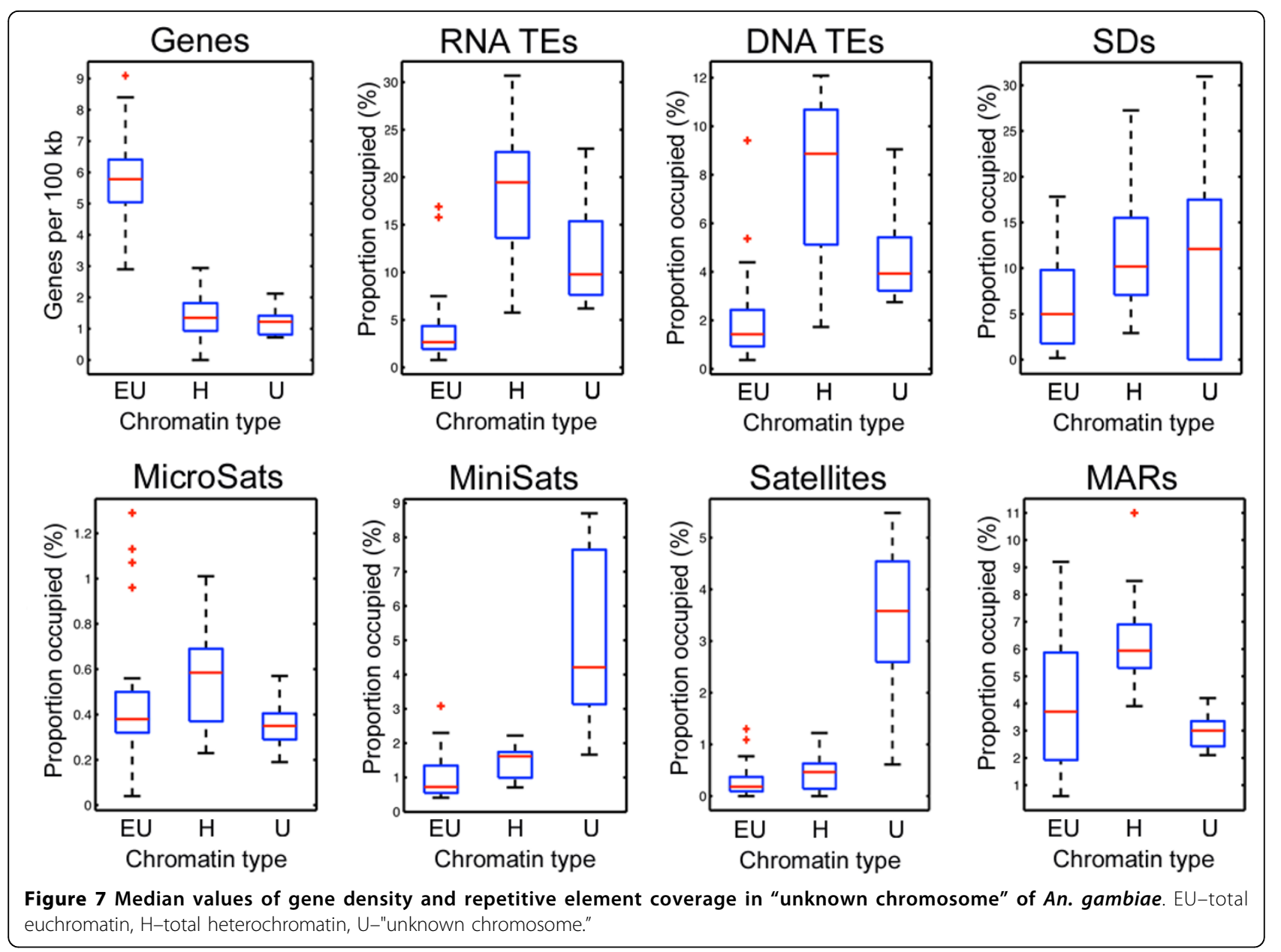

232 protein-coding genes is available for further characterization. GO analysis revealed that heterochromatin is enriched in genes that encode for proteins that may be involved in epigenetic regulation of chromatin. This study described the large regions of intercalary heterochromatin with a morphology not seen in D. melanogaster. We also provided evidence that heterochromatin and euchromatin significantly differ in gene density and the coverage of RNA TEs and SDs. The sequence composition, in terms of DNA TEs, RNA TEs, minisatellites, and satellites, can differentiate between the diffuse and compact types of intercalary heterochromatin. Conversely, MARs are distributed regardless of the chromatin type. The results of immunostaining with HP1 and lamin confirmed the general principle of nuclear organization-that the gene-poor regions of the genome reside at the nuclear periphery. Future investigations of An. gambiae heterochromatin need to show whether specific molecular composition can actually lead to chromosome-NE interactions. Given that the $42-\mathrm{Mb}-$ long "unknown chromosome" has the molecular characteristics of heterochromatin, it is possible that only one third of heterochromatic sequences in the An. gambiae genome assembly have been placed to chromosomes. Finally, we found that pericentric islands of genomic divergence between $\mathrm{M}$ and $\mathrm{S}$ incipient species of An. gambiae are almost completely heterochromatic, demonstrating the elevated evolutionary plasticity of the mosquito heterochromatin.

\section{Methods}

Mosquito strain and chromosome preparation

A laboratory SUA strain of An. gambiae was used in this study. Mosquitoes were reared at $28^{\circ} \mathrm{C}$ at $80 \%$ humidity. Mosquitoes were grown at a low density (500750 mosquitoes per 4 liter pan) to obtain better quality chromosomes. Larvae were fed ad libitum. Adults were given sugar water through dampened cotton balls that were removed at least 2 hours preblood feeding to ensure that most mosquitoes would take a blood meal. To obtain the chromosomal preparations, females were blood fed twice with a Guinea pig. Chromosomal slides for the morphological analysis were prepared as described previously [81]. Images were recorded with an 
Olympus Q-color5 digital cooled 5 megapixel camera and the Olympus CX41 light microscope using 1000x magnification (Olympus America Inc., Melville, NY, USA).

\section{Probe preparation and FISH}

Genomic DNA from An. gambiae mosquitoes was isolated via a DNeasy Blood and Tissue Kit (Qiagen Inc., Valencia, CA, USA). PCR probes were chosen from the euchromatin-heterochromatin transition zones of the An. gambiae genome. Many of these probes were based on genes located near expected heterochromatineuchromatin boundaries on each chromosome arm. Primers were designed using the Primer3 program [82]. PCR products ranged from $400-600 \mathrm{bp}$ in size. The in situ hybridization procedure was done as previously described [81]. PCR products were gel purified using the Geneclean kit (Qbiogene, Inc., Irvine, CA). The DNA was labeled with Cy3-AP3-dUTP (GE Healthcare UK Ltd., Buckinghamshire, England) using the Random Primer DNA Labeling System (Invitrogen Corporation, Carlsbad, CA, USA). DNA probes were hybridized to the chromosomes at $39^{\circ} \mathrm{C}$ overnight in hybridization solution (Invitrogen Corporation, Carlsbad, CA, USA). Then the chromosomes were washed in $0.2 \times \mathrm{SSC}$, (Saline-Sodium Citrate: 0.03 M Sodium Chloride, $0.003 \mathrm{M}$ Sodium Citrate) counterstained with YOYO-1, and mounted in DABCO. Fluorescent signals were detected and recorded using a Zeiss LSM 510 Laser Scanning Microscope (Carl Zeiss MicroImaging Inc., Thornwood, NY, USA).

\section{HP1 and lamin antibodies immunolocalization}

The original method of chromosome immunostaining was slightly modified for application to ovarian nurse cell polytene chromosomes $[64,83]$. In order to obtain polytene chromosomes from ovarian nurse cells, we blood fed female mosquitoes and kept them at regular conditions (temperature $26^{\circ} \mathrm{C}$, humidity $80 \%$ ) over night for 25 hours. Then half gravid females were placed on ice, and their ovaries were dissected. Every ovary was divided into two parts; each part was placed in fixative solution ( $47 \%$ water, $45 \%$ acetic acid, and $8 \%$ formaldehyde) separately; and follicles were spread on the slide by needles. Afterwards, the fixative solution was removed by filter paper, and follicles were placed in a fresh drop of the solution. Follicles were squashed under a cover slip and frozen in liquid nitrogen. Then cover slips were removed, and slides were kept in $70 \%$ cold ethanol at $-20^{\circ} \mathrm{C}$ for several hours. Just before immunohybridization, slides were washed in PBS saline buffer (Boston Bioproduct, Worcester, MA, USA) with $0.1 \%$ Nonidet P40 and incubated for 20 minutes in blocking solution (1\% BSA in PBS).
Primary mouse monoclonal antibodies C1A9 for Heterochromatin Protein 1 of D. melanogaster and ADL67.10 for Drosophila lamin $\mathrm{Dm}_{0}$ (Developmental Studies Hybridoma Bank, The University of Iowa, USA) were used for immunostaining of An. gambiae polytene chromosomes. Primary antibodies were diluted in 1:50 ratio and incubated overnight with the chromosomes in a humid chamber at $4^{\circ} \mathrm{C}$. Secondary goat antibodies to mouse were Cy3 labeled (KPL, Guildford, UK) and diluted in 1:200 ratio. Slides were incubated with secondary antibodies for 40 minutes at room temperature. Chromosomes were counterstained with YOYO-1 (Invitrogen, Way Carlsbad, CA 92008 USA) and mounted in DABCO antifade solution $(0.233 \mathrm{~g}$ DABCO, $800 \mu \mathrm{l}$ $\mathrm{H}_{2} \mathrm{O}, 200 \mu \mathrm{l} 1 \mathrm{M}$ trisHCl pH 8.0, $9 \mathrm{ml}$ glycerol). Slides were examined using a Zeiss LSM 510 Laser Scanning Microscope (Carl Zeiss MicroImaging Inc., Thornwood, NY, USA).

\section{GO annotation of heterochromatin and unmapped genome assembly}

The An. gambiae AgamP4 annotated peptide set was analyzed using a locally installed copy of Interproscan 4.4.1 [84]. A GO [75] annotation file was generated using Interproscan-assigned GO terms and custom Perl scripts. Go-Term-Finder [85] version 0.86 was used to search for significantly overrepresented (i.e., $\mathrm{p}<0.05$ ) $\mathrm{GO}$ terms assigned to genes in heterochromatin relative to frequencies for all GO-annotated genes in the peptide dataset. All scores reported have been Bonferroni corrected to account for multiple comparisons. Genes within the euchromatin-heterochromatin transition zones were considered euchromatic for this analysis. Bar graphs were generated with Microsoft Excel and labeled using Adobe Illustrator CS4.

\section{Gene and repetitive element databases}

Counts and length of coverage of all molecular features were identified in 5-Mb intervals in euchromatin and $<1-\mathrm{Mb}$ intervals in heterochromatin of the An. gambiae AgamP3 genome assembly [76]. Gene density and TE coverage were analyzed using the Biomart [86] and RepeatMasker [87] (http://www.repeatmasker.org/) programs, respectively. Micro- and minisatellites were analyzed by Tandem Repeats Finder [88]. Only tandem repeats with $80 \%$ matches and a copy number of 2 or more ( 8 or more for microsatellites) were included in the analysis. Microsatellites, minisatellites, and satellites had period sizes ranging from 2 to 6 , from 7 to 99 , and from 100 or more, respectively. SDs were detected using BLAST-based whole-genome assembly comparison [89] limited to putative SDs represented by pair-wise alignments with $\geq 2.5-\mathrm{kb}$ and $>90 \%$ sequence identity. The alignment length was specifically chosen to avoid the 
vast majority of incompletely masked repetitive elements. SD counts are not discrete duplication events, but indicate the number of regions that have been involved in duplications within a given interval. Putative MARs in the An. gambiae genome sequence were predicted using the SMARTest bioinformatic tool [90].

\section{Bayesian statistical analysis of molecular features in the chromatin types}

We have developed a model and procedure for discerning differences in molecular features between chromatin types. For this analysis, we incorporated data which distinguishes both the counts for each molecular feature and the overall coverage of each feature in subdivided regions of each of the five chromatin types of interest $\xi_{i}$ $\in A=\{\mathrm{EU}, \mathrm{PH}, \mathrm{IHc}, \mathrm{PEU}, \mathrm{IHd}\}$, where $\mathrm{PH}$-pericentric heterochromatin, IHc-compact intercalary heterochromatin, IHd-diffuse intercalary heterochromatin, PEUproximal euchromatin, EU-euchromatin. Since each region of the genome where these chromatin types are located is closely independent of each other, the likelihood follows as:

$$
\prod_{\xi_{j} \in A} \prod_{i \in \xi_{j}} \operatorname{Pr}\left(C_{i, \xi_{j}} \mid \text { Data, } \Theta\right)
$$

where $C_{i, \xi_{j}}$ are the counts associated with arm $\xi_{j}$ for chromatin type $i$ and $\Theta$ are the unknown model parameters that must be estimated.

For our application, we used a Poisson random effects model for explaining the counts, but included information about the coverage in each region as well. To make this connection, we parameterized the mean effect $\lambda_{i, \xi_{j}}$ through the log-link function as:

$$
\log \left(\lambda_{i, \xi_{j}}\right)=\mu_{\xi_{i}}+\beta_{\xi_{i}} \log \left(L_{i}\right)+\zeta_{\xi_{i}} \log \left(K_{i}\right)
$$

where $L_{i}$ is the total length and $K_{i}$ is the coverage length for chromatin type $i$. For each chromatin type, $\beta_{\xi_{i}}$ and $\zeta_{\xi_{i}}$ are random effects relating to the effect each length has on distinguishing the number of the molecular feature. $\mu_{\xi_{i}}$ relates to the overall density of the counts for each chromatin region. Hence in our case, the model unknowns are $\Theta=\left\{\mu_{\xi_{i}}, \beta_{\xi_{i}}, \zeta_{\xi_{i}}\right\}$ for each $\xi_{i} \in A=\{\mathrm{EU}, \mathrm{PH}, \mathrm{IH}$ c, PEU, IH d $\}$.

Our ultimate goal was to determine if random effects $\left\{\mu_{\xi_{i}}, \beta_{\xi_{i}}, \zeta_{\xi_{i}}\right\}$ can be statistically distinguished between chromatin types. Dominant model selection procedures have the ability to compare all possible competing models and also to compensate for the number of parameters involved in each model. That is, if model fit is the objective, then all procedures will determine optimality by utilizing as many parameters as is possible. In our case, these could correspond to 125 possible parameter configurations. Since models selected this way are generally suboptimal in terms of prediction, likelihood penalization schemes are common practice. For instance, the Bayesian information criterion (BIC) and the Akaike information criterion (AIC) are commonly used devices for selecting between models. We relied on the former, since this criterion closely aligns with Bayes Factor computation. Explicitly, BIC, under model $M_{k}$, is computed as:

$$
-B I C_{M_{k}}=2 \log (p(\text { Data } \mid \hat{M}))-\log (N) p
$$

where $p($ Data $\mid \hat{M})$ is the maximum likelihood estimate (MLE), under model $k, N$ is the number of observations, and $p$ is the number of parameters in model $k$.

Bayes factors select between models through the ratio

$$
B F=\frac{\int p\left(\text { Data } \mid \Theta, M_{k}\right) p\left(\Theta \mid M_{k}\right) d \Theta}{\int p\left(\text { Data } \mid \Theta, M_{\tilde{k}}\right) p\left(\Theta \mid M_{\tilde{k}}\right) d \Theta}
$$

which can be interpreted as the level of support model $M_{k}$ has in favor of the data over model $M_{\tilde{k}}$. As an approximation, we have

$$
e^{-\frac{1}{2} \Delta B I C}=e^{-\frac{1}{2}\left(B I C_{M_{k}}-B I C_{M_{\tilde{k}}}\right)} \approx B F
$$

which is a measure that decides between models and accounts for high degrees of observational variation. In order to compute the MLEs used in BIC calculations, we relied on an annealing algorithm. Specifically, given multiple locations in model space, state values in $\Theta$ and model configurations are simultaneously maximized to provide the MLE estimates for each data set. This procedure was repeated 1,000,000 times to ensure global optimization was achieved and that the best models (MAX models) were selected. The MAX models for each feature are given below.

RNA TEs: MAX model is $(\mathrm{PEU}=\mathrm{EU})-\mathrm{BIC}=$ -2471.87, $(\mathrm{PEU}=\mathrm{EU}, \mathrm{IHc}=\mathrm{IHd})-\mathrm{BIC}=-2474.12$, and all different $-(-\mathrm{BIC}=-2475.64)$. So, $\mathrm{PEU}=\mathrm{EU}$ has strong support (MAX model) over the model with all distinguishing chromatin types $\triangle \mathrm{BIC}=3.77$, and $\triangle \mathrm{BIC}$ $=1.52$ for distinguishing models with all distinct from $(\mathrm{PEU}=\mathrm{EU}, \mathrm{IHc}=\mathrm{IHd})$, which is moderate support that euchromatin and intercalary heterochromatin types can be considered the same for retroelements. All other models have negligible support $(\triangle \mathrm{BIC}>10)$.

DNA TEs: MAX model is $(\mathrm{IHc}=\mathrm{PEU})-\mathrm{BIC}=$ -1032.5, $(\mathrm{IHc}=\mathrm{PEU}, \mathrm{IHd}=\mathrm{PH})-\mathrm{BIC}=-1034.0$, so there is support for $(\mathrm{IHc}=\mathrm{PEU}, \mathrm{IHd}=\mathrm{PH}) \Delta \mathrm{BIC}=1.5$. All other hypotheses $\triangle \mathrm{BIC}>9$. 
SDs: MAX model is $(\mathrm{IHc}=\mathrm{IHd})-\mathrm{BIC}=-1540.2$, all other hypotheses have $\triangle \mathrm{BIC}>8$.

MARs: MAX model is $(\mathrm{PH}=\mathrm{IHc})-\mathrm{BIC}=-1305.21$, $(\mathrm{PH}=\mathrm{IHc}=\mathrm{IHd})-\mathrm{BIC}=-1306.44,(\mathrm{PH}=\mathrm{IHc}=\mathrm{IHd}=$ $\mathrm{PEU})-\mathrm{BIC}=-1309.39$. So, distinguishing $\mathrm{IHd}$ from $(\mathrm{PH}=$ $\mathrm{IHc}$ ) has support $\triangle \mathrm{BIC}=1.23$, which is mild. $\mathrm{PEU}$ is sufficiently different from each of the other candidate hypotheses, so we deem $(\mathrm{PH}=\mathrm{IHc}=\mathrm{IHd})$. Differentiation from the all distinguishable model has $\triangle \mathrm{BIC}>10$.

Genes: MAX model is $(\mathrm{EU}=\mathrm{PEU}, \mathrm{PH}=\mathrm{IH} \mathrm{c}=\mathrm{IH}$ d) - BIC 468.96, $\triangle \mathrm{BIC}>10$ for all nonnested hypotheses.

Microsatellites: MAX model is $(\mathrm{IHc}=\mathrm{PEU})-\mathrm{BIC}=$ -1408.22, $(\mathrm{IHc}=\mathrm{PEU}=\mathrm{IHd})-\mathrm{BIC}=-1407.98, \Delta \mathrm{BIC}=$ 1.76. Supported hypothesis is $(\mathrm{IHc}=\mathrm{PEU}=\mathrm{IHd})$.

Minisatellites: MAX model is $(\mathrm{PEU}=\mathrm{IHc})-\mathrm{BIC}=$ -1887.03, $(\mathrm{EU}=\mathrm{PEU}=\mathrm{IHc})-\mathrm{BIC}=-1890.15 \Delta \mathrm{BIC}=$ 3.12, so supported hypothesis is $\mathrm{EU}=\mathrm{PEU}=\mathrm{IHc}$, and less parsimoniously $\mathrm{PEU}=\mathrm{IHc}$. All other hypotheses have $\triangle \mathrm{BIC}>10$.

Satellites: MAX model is $(\mathrm{IHc}=\mathrm{PEU})-\mathrm{BIC}=-656.78$, all other hypotheses have $\triangle \mathrm{BIC}>10$.

\section{List of abbreviations}

AIC: Akaike information criterion; BIC: Bayesian information criterion; BSA: bovine serum albumin; DABCO: 1,4-diazabicyclo[2.2.2] octane; EU: euchromatin; FISH: fluorescent in situ hybridization; GO: gene ontology; HP1: heterochromatin protein 1; IHc: compact intercalary heterochromatin; IHd: diffuse intercalary heterochromatin; lhr: lethal hybrid rescue; MAR: matrix attachment region; MLE: maximum likelihood estimate; MR4: Malaria Research and Reference Reagent Resource Center; NE: nuclear envelope; PBS: phosphate buffered saline; PH: pericentric heterochromatin; PEU: proximal euchromatin; SD: segmental duplication; SSC: salinesodium citrate; TE: transposable element.

\section{Additional material}

Additional file 1: Table S1. Coverage (\%) of molecular elements in chromatin types of An. gambiae.

\begin{abstract}
Acknowledgements
The SUA colony of An. gambiae was obtained from the Malaria Research and Reference Reagent Resource Center (MR4). We thank Melissa Wade for editing the text and Mike Wong and the SFSU Center for Computing for Life Sciences for technical assistance with software installation and hardware maintenance. This work was supported by startup funds from Virginia Tech and National Institutes of Health grants 5R21AI074729-02 and 1R21Al08102301 (to I.V.S) and 5R01HG000747-14 (to C.D.S).
\end{abstract}

\section{Author details}

'Department of Entomology, Virginia Tech, Blacksburg, VA 24061, USA. ${ }^{2}$ Department of Molecular and Cellular Biology, Institute of Chemical Biology and Fundamental Medicine, Siberian Branch of Russian Academy of Sciences, Novosibirsk 630090, Russia. ${ }^{3}$ Department of Statistics, Virginia Tech,

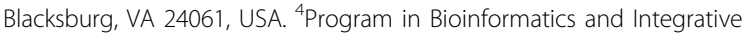
Biology and Department of Medicine, Division of Transfusion Medicine, University of Massachusetts Medical School, Worcester, MA 01605, USA.

${ }^{5}$ Department of Biology, San Francisco State University, San Francisco, CA 94132, USA. ${ }^{6}$ Drosophila Heterochromatin Genome Project, Lawrence Berkeley National Lab, Berkeley, CA 94720, USA

\section{Authors' contributions}

IVS designed research; MVS, PG, IVB, SCL, JAB, CDS, and IVS performed research; $S C L$ and $J A B$ contributed new reagents/analytic tools; MVS, PG, IVB, $S C L, J A B, C D S$, and IVS analyzed data; and MVS, IVS and SCL wrote the paper. All authors read and approved the final manuscript.

Received: 26 April 2010 Accepted: 4 August 2010

Published: 4 August 2010

\section{References}

1. Bernard P, Maure JF, Partridge JF, Genier S, Javerzat JP, Allshire RC: Requirement of heterochromatin for cohesion at centromeres. Science 2001, 294:2539-2542

2. Dernburg AF, Sedat JW, Hawley RS: Direct evidence of a role for heterochromatin in meiotic chromosome segregation. Cell 1996, 86:135-146.

3. Swedlow JR, Lamond Al: Nuclear dynamics: where genes are and how they got there. Genome Biol 2001, 2:REVIEWS0002.

4. Zhimulev IF: Polytene chromosomes, heterochromatin, and position effect variegation. Adv Genet 1998, 37:1-566.

5. Riddle NC, Elgin SC: A role for RNAi in heterochromatin formation in Drosophila. Curr Top Microbiol Immunol 2008, 320:185-209.

6. Vermaak D, Malik HS: Multiple roles for heterochromatin protein 1 genes in Drosophila. Annu Rev Genet 2009, 43:467-492.

7. Girton JR, Johansen KM: Chromatin structure and the regulation of gene expression: the lessons of PEV in Drosophila. Adv Genet 2008, 61:1-43.

8. Abad P, Vaury C, Pelisson A, Chaboissier MC, Busseau I, Bucheton A: A long interspersed repetitive element-the I factor of Drosophila teissieri-is able to transpose in different Drosophila species. Proc Natl Acad Sci USA 1989, 86:8887-8891.

9. Weiler KS, Wakimoto BT: Heterochromatin and gene expression in Drosophila. Annu Rev Genet 1995, 29:577-605.

10. Hoskins RA, Smith CD, Carlson JW, Carvalho AB, Halpern A, Kaminker JS, Kennedy C, Mungall CJ, Sullivan BA, Sutton GG, et al: Heterochromatic sequences in a Drosophila whole-genome shotgun assembly. Genome Biol 2002, 3:RESEARCH0085

11. Hoskins RA, Carlson JW, Kennedy C, Acevedo D, Evans-Holm M, Frise E, Wan KH, Park S, Mendez-Lago M, Rossi F, et al: Sequence finishing and mapping of Drosophila melanogaster heterochromatin. Science 2007, 316:1625-1628.

12. Smith CD, Shu S, Mungall CJ, Karpen GH: The Release 5.1 annotation of Drosophila melanogaster heterochromatin. Science 2007, 316:1586-1591.

13. Andreyeva EN, Kolesnikova TD, Demakova OV, Mendez-Lago M, Pokholkova GV, Belyaeva ES, Rossi F, Dimitri P, Villasante A, Zhimulev IF: High-resolution analysis of Drosophila heterochromatin organization using SuUR Su(var)3-9 double mutants. Proc Natl Acad Sci USA 2007, 104:12819-12824.

14. Fitzpatrick KA, Sinclair DA, Schulze SR, Syrzycka M, Honda BM: A genetic and molecular profile of third chromosome centric heterochromatin in Drosophila melanogaster. Genome/National Research Council Canada = Genome/Conseil national de recherches Canada 2005, 48:571-584.

15. Prokofyeva-Belgovskaya AA: Inert regions of internal parts in Xchromosome Drosophila melanogaster. Proc Acad SCi USSR 1939, 3:362-370

16. Zhimulev IF, Belyaeva ES, Makunin IV, Pirrotta V, Semeshin VF, Alekseyenko AA, Belyakin SN, Volkova El, Koryakov DE, Andreyeva EN, et al: Intercalary heterochromatin in Drosophila melanogaster polytene chromosomes and the problem of genetic silencing. Genetica 2003, 117:259-270.

17. Belyakin SN, Christophides GK, Alekseyenko AA, Kriventseva EV, Belyaeva ES, Nanayev RA, Makunin IV, Kafatos FC, Zhimulev IF: Genomic analysis of Drosophila chromosome underreplication reveals a link between replication control and transcriptional territories. Proc Natl Acad Sci USA 2005, 102:8269-8274. 
18. Heitz E: Uber $\alpha$ - und $\beta$-Heterochromatin sowie Konstanz und Bau der Chromomeren bei Drosophila. Biol Zbl 1934, 54:588-609.

19. Vaury C, Bucheton A, Pelisson A: The beta heterochromatic sequences flanking the I elements are themselves defective transposable elements. Chromosoma 1989, 98:215-224.

20. Miklos GL, Yamamoto MT, Davies J, Pirrotta V: Microcloning reveals a high frequency of repetitive sequences characteristic of chromosome 4 and the beta-heterochromatin of Drosophila melanogaster. Proc Natl Acad Sci USA 1988, 85:2051-2055.

21. Strahl $B D$, Allis $C D$ : The language of covalent histone modifications. Nature 2000, 403:41-45.

22. Bannister AJ, Zegerman P, Partridge JF, Miska EA, Thomas JO, Allshire RC, Kouzarides T: Selective recognition of methylated lysine 9 on histone $\mathrm{H} 3$ by the HP1 chromo domain. Nature 2001, 410:120-124.

23. Lachner M, O'Carroll D, Rea S, Mechtler $K$, Jenuwein T: Methylation of histone H3 lysine 9 creates a binding site for HP1 proteins. Nature 2001, 410:116-120.

24. James TC, Elgin SCR: Identification of a nonhistone chromosomal protein associated with heterochromatin in Drosophila melanogaster and its gene. Molecular and Cellular Biology 1986, 6:3862-3872.

25. Riddle NC, Elgin SC: The dot chromosome of Drosophila: insights into chromatin states and their change over evolutionary time. Chromosome Res 2006, 14:405-416.

26. Riddle NC, Leung W, Haynes KA, Granok H, Wuller J, Elgin SC: An investigation of heterochromatin domains on the fourth chromosome of Drosophila melanogaster. Genetics 2008, 178:1177-1191.

27. Slawson EE, Shaffer CD, Malone CD, Leung W, Kellmann E, Shevchek RB, Craig CA, Bloom SM, Bogenpohl J, Dee J, et al: Comparison of dot chromosome sequences from $D$. melanogaster and $D$. virilis reveals an enrichment of DNA transposon sequences in heterochromatic domains. Genome Biol 2006, 7:R15.

28. Baricheva EA, Berrios M, Bogachev SS, Borisevich IV, Lapik ER, Sharakhov IV, Stuurman N, Fisher PA: DNA from Drosophila melanogaster betaheterochromatin binds specifically to nuclear lamins in vitro and the nuclear envelope in situ. Gene 1996, 171:171-176.

29. Hochstrasser M, Sedat JW: Three-dimensional organization of Drosophila melanogaster interphase nuclei. II. Chromosome spatial organization and gene regulation. J Cell Biol 1987, 104:1471-1483.

30. Hochstrasser M, Sedat JW: Three-dimensional organization of Drosophila melanogaster interphase nuclei. I. Tissue-specific aspects of polytene nuclear architecture. J Cell Biol 1987, 104:1455-1470.

31. Akhtar A, Gasser SM: The nuclear envelope and transcriptional control. Nat Rev Genet 2007, 8:507-517.

32. Scherthan $\mathrm{H}$ : Telomere attachment and clustering during meiosis. Cell Mol Life Sci 2007, 64:117-124

33. Singh PB, Georgatos SD: HP1: facts, open questions, and speculation. J Struct Biol 2002, 140:10-16.

34. Hochstrasser M, Mathog D, Gruenbaum Y, Saumweber H, Sedat JW: Spatial organization of chromosomes in the salivary gland nuclei of Drosophila melanogaster. J Cell Biol 1986, 102:112-123.

35. Stegnii VN, Sharakhova MV: Systemic reorganization of the architechtonics of polytene chromosomes in onto- and phylogenesis of malaria mosquitoes. Structural features regional of chromosomal adhesion to the nuclear membrane. Genetika 1991, 27:828-835.

36. Sharakhov IV, Sharakhova MV, Mbogo CM, Koekemoer LL, Yan G: Linear and spatial organization of polytene chromosomes of the African malaria mosquito Anopheles funestus. Genetics 2001, 159:211-218.

37. Rzepecki R, Bogachev SS, Kokoza E, Stuurman N, Fisher PA: In vivo association of lamins with nucleic acids in Drosophila melanogaster. J Cell Sci 1998, 111(Pt 1):121-129.

38. Stierle V, Couprie J, Ostlund C, Krimm I, Zinn-Justin S, Hossenlopp P, Worman HJ, Courvalin JC, Duband-Goulet I: The carboxyl-terminal region common to lamins $\mathrm{A}$ and $\mathrm{C}$ contains a DNA binding domain. Biochemistry 2003, 42:4819-4828.

39. Dechat T, Pfleghaar K, Sengupta K, Shimi T, Shumaker DK, Solimando L, Goldman RD: Nuclear lamins: major factors in the structural organization and function of the nucleus and chromatin. Genes Dev 2008, 22:832-853.

40. Luderus ME, de Graaf A, Mattia E, den Blaauwen JL, Grande MA, de Jong L, van Driel R: Binding of matrix attachment regions to lamin B1. Cell 1992, 70:949-959.
41. Strausbaugh LD, Williams SM: High density of an SAR-associated motif differentiates heterochromatin from euchromatin. J Theor Biol 1996, 183:159-167.

42. von Sternberg R, Shapiro JA: How repeated retroelements format genome function. Cytogenet Genome Res 2005, 110:108-116.

43. Shapiro JA, von Sternberg R: Why repetitive DNA is essential to genome function. Biol Rev Camb Philos Soc 2005, 80:227-250.

44. Holt RA, Subramanian GM, Halpern A, Sutton GG, Charlab R, Nusskern DR, Wincker P, Clark AG, Ribeiro JM, Wides $R$, et al: The genome sequence of the malaria mosquito Anopheles gambiae. Science 2002, 298:129-149.

45. Gatti M, Santini G, Pimpinelli S, Coluzz M: Fluorescence banding techniques in the identification of sibling species of the anopheles gambiae complex. Heredity 1977, 38:105-108.

46. Sharakhova MV, Stegnii VN, Braginets OP: Interspecies differences in the ovarian trophocyte precentromere heterochromatin structure and evolution of the malaria mosquito complex Anopheles maculipennis. Genetika 1997, 33:1640-1648.

47. Sharakhova MV, Stegnii VN, Timofeeva OV: Polymorphism of pericentromere heterochromatin of polytene chromosomes of ovarian trophocytes in natural populations of the malaria mosquito Anopheles messeae Fall. Genetika 1997, 33:281-283.

48. Bonaccorsi S, Santini G, Gatti M, Pimpinelli S, Colluzzi M: Intraspecific polymorphism of sex chromosome heterochromatin in two species of the Anopheles gambiae complex. Chromosoma 1980, 76:57-64.

49. Fraccaro M, Tiepolo L, Laudani U, Marchi A, Jayakar SD: Y chromosome controls mating behaviour on Anopheles mosquitoes. Nature 1977, 265:326-328.

50. della Torre A, Merzagora L, Powell JR, Coluzzi M: Selective introgression of paracentric inversions between two sibling species of the Anopheles gambiae complex. Genetics 1997, 146:239-244.

51. Lehmann T, Diabate A: The molecular forms of Anopheles gambiae: a phenotypic perspective. Infect Genet Evol 2008, 8:737-746.

52. Turner TL, Hahn MW, Nuzhdin SV: Genomic islands of speciation in Anopheles gambiae. PLoS Biol 2005, 3:e285.

53. White BJ, Cheng C, Simard F, Costantini C, Besansky NJ: Genetic association of physically unlinked islands of genomic divergence in incipient species of Anopheles gambiae. Mol Ecol 2010, 19:925-939.

54. Besansky NJ, Powell JR: Reassociation kinetics of Anopheles gambiae (Diptera: Culicidae) DNA. J Med Entomol 1992, 29:125-128.

55. Sharakhova M, Hammond MP, Lobo NF, Krzywinski J, Unger MF, Hillenmeyer ME, Bruggner RV, Birney E, Collins FH: Update of the Anopheles gambiae PEST genome assembly. Genome Biol 2007, 8:R5.

56. Sharakhova MV, Sharakhov IV, Gosteva LG, Kotlikova IV, Stegnii VN: Pericentromeric and intercalary alpha-heterochromatin of polytene chromosomes of the malaria mosquito. Genetika 2000, 36:175-181.

57. Belyaeva ES, Demakov SA, Pokholkova GV, Alekseyenko AA, Kolesnikova TD, Zhimulev IF: DNA underreplication in intercalary heterochromatin regions in polytene chromosomes of Drosophila melanogaster correlates with the formation of partial chromosomal aberrations and ectopic pairing. Chromosoma 2006, 115:355-366.

58. Mal'ceva NI, Gyurkovics H, Zhimulev IF: General characteristics of the polytene chromosome from ovarian pseudonurse cells of the Drosophila melanogaster otu11 and fs(2)B mutants. Chromosome Res 1995, 3:191-200.

59. James TC, Eissenberg JC, Craig C, Dietrich V, Hobson A, Elgin SC: Distribution patterns of HP1, a heterochromatin-associated nonhistone chromosomal protein of Drosophila. Eur J Cell Biol 1989, 50:170-180.

60. Buglia GL, Ferraro M: Germline cyst development and imprinting in male mealybug Planococcus citri. Chromosoma 2004, 113:284-294.

61. Fanti L, Berloco M, Piacentini L, Pimpinelli S: Chromosomal distribution of heterochromatin protein 1 (HP1) in Drosophila: a cytological map of euchromatic HP1 binding sites. Genetica 2003, 117:135-147.

62. Piacentini L, Fanti L, Berloco M, Perrini B, Pimpinelli S: Heterochromatin protein 1 (HP1) is associated with induced gene expression in Drosophila euchromatin. J Cell Biol 2003, 161:707-714.

63. Cryderman DE, Grade SK, Li Y, Fanti L, Pimpinelli S, Wallrath LL: Role of Drosophila HP1 in euchromatic gene expression. Dev Dyn 2005, 232:767-774

64. Koryakov DE, Reuter G, Dimitri P, Zhimulev IF: The SuUR gene influences the distribution of heterochromatic proteins HP1 and SU(VAR)3-9 on nurse cell polytene chromosomes of Drosophila melanogaster. 
Chromosoma 2006, 115:296-310.

65. Aasland R, Stewart AF: The chromo shadow domain, a second chromo domain in heterochromatin-binding protein 1, HP1. Nucleic Acids Res 1995, 23:3168-3173.

66. Lomberk G, Wallrath $L$, Urrutia R: The Heterochromatin Protein 1 family. Genome Biol 2006, 7:228.

67. Grewal SI, Elgin SC: Transcription and RNA interference in the formation of heterochromatin. Nature 2007, 447:399-406.

68. Ye Q, Worman $\mathrm{HJ}$ : Interaction between an integral protein of the nuclear envelope inner membrane and human chromodomain proteins homologous to Drosophila HP1. J Biol Chem 1996, 271:14653-14656.

69. Ye Q, Callebaut I, Pezhman A, Courvalin JC, Worman HJ: Domain-specific interactions of human HP1-type chromodomain proteins and inner nuclear membrane protein LBR. J Biol Chem 1997, 272:14983-14989.

70. Pickersgill H, Kalverda B, de Wit E, Talhout W, Fornerod M, van Steensel B: Characterization of the Drosophila melanogaster genome at the nuclear lamina. Nat Genet 2006, 38:1005-1014.

71. Wang-Sattler R, Blandin S, Ning Y, Blass C, Dolo G, Toure YT, Torre AD, Lanzaro GC, Steinmetz LM, Kafatos FC, Zheng L: Mosaic genome architecture of the Anopheles gambiae species complex. PLOS ONE 2007, 2:e1249.

72. Vermaak D, Henikoff $S$, Malik HS: Positive selection drives the evolution of rhino, a member of the heterochromatin protein 1 family in Drosophila. PLoS Genet 2005, 1:96-108.

73. Talbert PB, Bryson TD, Henikoff S: Adaptive evolution of centromere proteins in plants and animals. $J$ Biol 2004, 3:18.

74. Brideau NJ, Flores HA, Wang J, Maheshwari S, Wang X, Barbash DA: Two Dobzhansky-Muller genes interact to cause hybrid lethality in Drosophila. Science 2006, 314:1292-1295.

75. Ashburner M, Ball CA, Blake JA, Botstein D, Butler H, Cherry JM, Davis AP, Dolinski K, Dwight SS, Eppig JT, et al: Gene ontology: tool for the unification of biology. The Gene Ontology Consortium. Nat Genet 2000, 25:25-29.

76. Lawson D, Arensburger P, Atkinson P, Besansky NJ, Bruggner RV, Butler R, Campbell KS, Christophides GK, Christley S, Dialynas E, et al: VectorBase: a data resource for invertebrate vector genomics. Nucleic Acids Res 2009, 37:D583-587.

77. Krzywinski J, Sangare D, Besansky NJ: Satellite DNA from the $Y$ chromosome of the malaria vector Anopheles gambiae. Genetics 2005, 169:185-196.

78. Redfern CP: Satellite DNA of Anopheles stephensi Liston (Diptera: Culicidae). Chromosomal location and under-replication in polytene nuclei. Chromosoma 1981, 82:561-581.

79. Biessmann H, Donath J, Walter MF: Molecular characterization of the Anopheles gambiae $2 \mathrm{~L}$ telomeric region via an integrated transgene. Insect Mol Biol 1996, 5:11-20.

80. Biessmann H, Kobeski F, Walter MF, Kasravi A, Roth CW: DNA organization and length polymorphism at the $2 \mathrm{~L}$ telomeric region of Anopheles gambiae. Insect Mol Biol 1998, 7:83-93.

81. Sharakhova MV, Xia A, McAlister SI, Sharakhov IV: A standard cytogenetic photomap for the mosquito Anopheles stephensi (Diptera: Culicidae): application for physical mapping. J Med Entomol 2006, 43:861-866.

82. Rozen S, Skaletsky H: Primer3 on the www for general users and for biologist programmers. Methods Mol Biol 2000, 132:365-386.

83. Stephens GE, Craig CA, Li Y, Wallrath LL, Elgin SC: Immunofluorescent staining of polytene chromosomes: exploiting genetic tools. Methods Enzymol 2004, 376:372-393.

84. Zdobnov EM, Apweiler R: InterProScan-an integration platform for the signature-recognition methods in InterPro. Bioinformatics 2001, 17:847-848.

85. Boyle El, Weng S, Gollub J, Jin H, Botstein D, Cherry JM, Sherlock G: GO: TermFinder-open source software for accessing Gene Ontology information and finding significantly enriched Gene Ontology terms associated with a list of genes. Bioinformatics 2004, 20:3710-3715.

86. Haider S, Ballester B, Smedley D, Zhang J, Rice P, Kasprzyk A: BioMart Central Portal-unified access to biological data. Nucleic Acids Res 2009, 37:W23-27.

87. Smit AFA, Hubley R, Green P: (1996-2004) RepeatMasker Open-3.0. [http:// www.repeatmasker.org/].

88. Benson G: Tandem repeats finder: a program to analyze DNA sequences. Nucleic Acids Res 1999, 27:573-580.
89. Bailey JA, Yavor AM, Massa HF, Trask BJ, Eichler EE: Segmental duplications: organization and impact within the current human genome project assembly. Genome Res 2001, 11:1005-1017.

90. Frisch M, Frech $\mathrm{K}$, Klingenhoff A, Cartharius K, Liebich I, Werner T: In silico prediction of scaffold/matrix attachment regions in large genomic sequences. Genome Res 2002, 12:349-354.

91. George P, Sharakhova M, Sharakhov I: High-resolution cytogenetic map for the African malaria vector Anopheles gambiae. Insect Mol Biol 2010.

doi:10.1186/1471-2164-11-459

Cite this article as: Sharakhova et al:: Genome mapping and characterization of the Anopheles gambiae heterochromatin. BMC Genomics 2010 11:459.

\section{Submit your next manuscript to BioMed Central and take full advantage of:}

- Convenient online submission

- Thorough peer review

- No space constraints or color figure charges

- Immediate publication on acceptance

- Inclusion in PubMed, CAS, Scopus and Google Scholar

- Research which is freely available for redistribution 